\title{
On Approximate Matrix Inversion Methods for Massive MIMO Detectors
}

\author{
Mahmoud A. M. Albreem and Ayman A. El-Saleh \\ A'Sharqiyah University \\ Ibra, Oman \\ Email: firstname.lastname@asu.edu.om
}

\author{
Markku Juntti \\ University of Oulu \\ Oulu, Finland \\ Email: firstname.lastname@oulu.fi
}

\begin{abstract}
Massive multiple-input multiple-output (MIMO) systems have been proposed to meet the user demands in terms of performance and quality of service $(\mathrm{QoS})$. Due to the large number of antennas, detectors in massive MIMO are playing a crucial role in guaranteeing a satisfactory performance, while their complexity is also being increased. This paper considers several approximate algorithms to avoid direct matrix inversion, namely the Neumann method, the Gauss-Seidel (GS) method, the successive over-relaxation (SOR) method, the Jacobi method, the Richardson method, the optimized coordinate descent (OCD), and the conjugate gradients (CG) method. Also, this paper presents a comparison among the approximate matrix inversion methods and the minimum mean square error (MMSE). Simulation of $16 \times 128$, and $16 \times 32$ MIMO systems shows that a detector based on the GS method outperforms other detectors when the ratio of base station (BS) antennas to user terminal antennas, $\beta$, is small. On the other hand, the detector based on the SOR method outperforms the other approximate matrix inversion methods when $\beta$ is large. In addition, this paper studies and recommends the setting values of relaxation parameter $(\omega)$ in the SOR and Richardson methods. It also provides a comparison among the approximate matrix inversion methods in the number of multiplications. Simulation results show that the Neumann method, the OCD method, and the CG method achieve the lowest number of multiplications while the CG method outperforms the Neumann and the OCD methods. This paper also shows that not every iteration improves the performance.
\end{abstract}

Index Terms-Massive MIMO, approximate matrix inversion, MMSE, detection

\section{INTRODUCTION}

Year-over-year, mobile data traffic is being increasingly growing. For instance, the mobile data traffic is expected to increase from 7.2 exabytes per month in 2016 to hit 49 exabytes per month by 2021 where $78 \%$ of the world's data traffic will be videos [1]. Therefore, the fourth generation (4G) communication systems require an essential improvement to meet the user's demand [2], [3]. Sequentially, fifth generation $(5 \mathrm{G})$ wireless communication is currently proposed with higher bandwidth, broader coverage, and ultra-low latency [3]. 5G will be driven largely by several technologies such as massive multiple-input multiple-output (MIMO), internet of things (IoT), millimeter wave (mmWave), device-to-device (D2D) communication and ultra dense networks (UDNs) [4].

MIMO has been successfully implemented in many wireless communication systems, such as the $3 \mathrm{G}$ and $4 \mathrm{G}$ where up to eight antenna elements have been deployed at the base station.
Massive MIMO is a multiuser communications system that employs a large number of antenna elements to serve simultaneously multiple users with a flexibility to opt what users to schedule for reception at any given time. The massive MIMO system increases the spatial multiplexing gain and the diversity gain by adding massive antennas at the base station (BS) to serve large number of users with relatively simple scheduling and receiver algorithms. However, numerous antennas create new challenges for signal processing in precoding [5], channel estimation [6] and signal detection [7].

Although maximum-likelihood (ML) detector achieves the optimum performance, it is unfeasible for massive MIMO systems because of its exponential complexity. In literature, suboptimal low complexity detectors have been proposed for signal detection in massive MIMO such as neighborhood search algorithms [8], lattice reduction algorithms [9], algorithms based on quadratic programming [10], sphere decoders [11]-[13], successive relaxation detection [14], successive interference cancellation (SIC) [15], graph models and belief propagation [16]. However, numerous antennas require a larger channel matrix to be considered by signal processing, which requires better algorithms and more powerful hardware chips in the physical layer. Matrix inversion is one of the main challenges for massive MIMO precoding and detection. Recently, approximate matrix inversion [14], [17]-[21] has drawn the attention of the research community for its capability of achieving a satisfactory balance between complexity and performance.

In this paper, a comparison between several approximate matrix inversion methods will be provided when the ratio of BS antennas to user terminal antennas $(\beta)$ varies. In addition, the contribution of the number of iterations $(n)$ in the performance-complexity profile will be discussed. The setting values of the relaxation parameter $(\omega)$ in SOR and Richardson methods will be studied and recommended. This paper also compares the performance-complexity profile among the approximate matrix inversion methods.

This paper is organized as follows: Section II presents the ML, MMSE, and detectors based approximate matrix inversion methods. Section III presents the results as well as the discussion. Section IV concludes the paper. 


\section{MASSIVE MIMO DETECTOR}

Massive MIMO is a multi-user system with $N$ antennas at the base-station (BS) availing $K$ single antenna users where $N \gg K$. Let $\mathbf{x}=\left[x_{1}, x_{2}, \ldots ., x_{K}\right]^{T}$ and $\mathbf{y}=\left[y_{1}, y_{2}, \ldots . ., y_{N}\right]^{T}$ where $\mathbf{x}$ and $\mathbf{y}$ vectors are the transmitted and the received signals respectively. Also $x_{K} \in C$ where $C$ is the modulation alphabet. Elements of the channel matrix $(\mathbf{H})$ are herein assumed to be independent and identically distributed (i.i.d) Gaussian random variables with zero mean and unit variance. The relationship between the input and the output of massive MIMO detector is represented mathematically in

$$
\mathbf{y}=\mathbf{H x}+\mathbf{n}
$$

where $\mathbf{n}$ is the $N \times 1$ additive white Gaussian noise (AWGN) whose entries are i.i.d. However, MIMO signal detection problem is NP-hard optimization problem [22]. An efficient massive MIMO system requires advanced signal detection techniques to achieve a satisfactory balance between the system performance and computational complexity. This section presents the concepts of ML, MMSE, and detectors based on the approximate matrix inversion methods.

\section{A. Maximum likelihood (ML)}

Maximum-likelihood (ML) detector achieves optimal performance but the complexity is exponential in the number of decision variables $|C|^{K}$. ML detector examines all possible signals as illustrated in

$$
\hat{\mathbf{x}}=\arg \min _{x \in|C|^{K}}\left\|\mathbf{y}-\sqrt{\frac{S N R}{K}} \mathbf{H x}\right\|^{2},
$$

where $\hat{\mathbf{x}}$ is the estimated received signal and SNR refers to the signal-to-noise ratio. ML detector is unfeasible for massive MIMO systems because it requires an exponential complexity. Therefore, it is mandatory to utilize suboptimal detectors with a reduced complexity in massive MIMO systems. A widely utilized suboptimal detector for massive MIMO is the MMSE.

\section{B. Minimum Mean Square Estimation (MMSE)}

MMSE detector minimizes the mean-square error (MSE) between the transmitted $\mathbf{x}$ and the estimated signal $\mathbf{H}^{H} \mathbf{y}$ [23]. The detected signal can be expressed as

$$
\hat{\mathbf{x}}_{M M S E}=\left(\mathbf{H}^{H} \mathbf{H}+\frac{K}{S N R} \mathbf{I}\right)^{-1} \mathbf{H}^{H} \mathbf{y} .
$$

MMSE algorithm (3) depends on reduced noise enhancement. However, the complexity of MMSE-based detector is still high for massive MIMO system because the detector involves matrix inversion and computation of Gram matrix with a complexity $O\left(N^{3}\right)$ and $O\left(N K^{2}\right)$, respectively [24]. Thus, approximate matrix inversion methods such as Neumman, GS, SOR, Jacobi, Richardson and OCD methods are needed to reduce the complexity.

\section{Neumann method}

In the Neumann method, the Gram matrix $\mathbf{G}=\mathbf{H}^{H} \mathbf{H}$ has been decomposed into $\mathbf{G}=\mathbf{D}+\mathbf{E}$, where $\mathbf{E}$ is the non-diagonal matrix and $\mathbf{D}$ is the main diagonal matrix [25]. The inverse of Gram matrix can be found as

$$
\mathbf{G}^{-1}=\sum_{i=0}^{\infty}\left(-\mathbf{D}^{-1} \mathbf{E}\right)^{i} \mathbf{D}^{-1}
$$

which converges to the matrix inverse $\mathbf{G}^{-1}$ if the condition

$$
\lim _{i \rightarrow \infty}\left(-\mathbf{D}^{-1} \mathbf{E}\right)^{i}=0
$$

is satisfied. In real systems, a sum of finite terms $(i)$ is utilized (4) and thus, a fixed number of iterations is performed.

A high precision of the matrix inverse will be achieved by increasing the number of iterations $(n)$ in expenses of higher computational complexity. This method suffers from considerable performance loss when the ratio between BS antennas and the user antennas, $\beta$, is close to 1 .

\section{Successive Over-Relaxation}

The detected signal using SOR iteration is described as

$\hat{\mathbf{x}}^{(n)}=\left(\frac{1}{\omega} \mathbf{D}+\mathbf{L}\right)^{-1}\left(\hat{\mathbf{x}}_{M F}+\left(\left(\frac{1}{\omega}-1\right) \mathbf{D}-\mathbf{U}\right) \hat{\mathbf{x}}^{(n-1)}\right)$,

where $\omega$ is the relaxation parameter and it plays a crucial role in the convergence and convergence rate. In SOR method, a Gram matrix should be pre-computed and provided as an input which increases the computational complexity [26]. It also has uncertain relaxation parameter $0<\omega<2$.

\section{E. Gauss-Seidel Method}

GS method is a special case of the SOR method where $\omega=1$. In GS method, any Hermitian positive definite matrix, A can be decomposed into $\mathbf{A}=\mathbf{D}+\mathbf{L}+\mathbf{U}$ [20] [27], where $\mathbf{D}, \mathbf{L}$ and $\mathbf{U}$ are the diagonal component, the strictly lower triangular component, and the strictly upper triangular component, respectively. It can be utilized to estimate the transmitted signal vector $(\hat{\mathbf{x}})[21]$ as

$$
\hat{\mathbf{x}}^{(n)}=(\mathbf{D}+\mathbf{L})^{-1}\left(\hat{\mathbf{x}}_{M F}-\mathbf{U} \hat{\mathbf{x}}^{(n-1)}\right), \quad n=1,2, \cdots,
$$

where $\hat{\mathbf{x}}_{M F}$ is the output of matched filtering method. The initial solution $\hat{\mathbf{x}}^{(0)}$, can be considered as zero if there is no priori information about its value [27]. It also requires an internal sequential iterations structure, thus, GS is not suitable for parallel implementation [20].

\section{F. Jacobi Method}

Jacobi method determines the solution of a diagonally dominant system as

$$
\hat{\mathbf{x}}^{(n)}=\mathbf{D}^{-1}\left(\hat{\mathbf{x}}_{M F}+(\mathbf{D}-\mathbf{A}) \hat{\mathbf{x}}^{(n-1)}\right),
$$

which holds if:

$$
\lim _{n \rightarrow \infty}\left(\mathbf{I}-\mathbf{D}^{-1} \mathbf{A}\right)^{n}=0 .
$$


The initial estimation can be identified as

$$
\hat{\mathbf{x}}^{(0)}=\mathbf{D}^{-1} \hat{\mathbf{x}}_{M F} .
$$

However, Jacobi method converges slowly, and thus, implying higher latency [28].

\section{G. Conjugate Gradients Method}

The estimated signal $(\hat{\mathbf{x}})$ can be obtained using

$$
\hat{\mathbf{x}}^{(n+1)}=\hat{\mathbf{x}}^{(n)}+\alpha^{(n)} \mathbf{p}^{(n)},
$$

where $\mathbf{p}^{(n)}$ is the conjugate direction [29] [30] with respect to A, i.e.

$$
\left(\mathbf{p}^{(n)}\right)^{H} \mathbf{A} \mathbf{p}^{(j)}=0, \quad \text { for } n \neq j
$$

and

$$
\mathbf{p}^{(n)}=\hat{\mathbf{x}}_{M F}^{(n)}+\frac{\hat{\mathbf{x}}_{M F}^{(n)} \cdot \hat{\mathbf{x}}_{M F}^{(n)}}{\hat{\mathbf{x}}_{M F}^{(n-1)} \cdot \hat{\mathbf{x}}_{M F}^{(n-1)}} \mathbf{p}^{(n-1)},
$$

and $\alpha^{(n)}$ is a scalar parameter shown as

$$
\alpha^{(n)}=\frac{\hat{\mathbf{x}}_{M F}^{(n)} \cdot \hat{\mathbf{x}}_{M F}^{(n)}}{\mathbf{A} \hat{\mathbf{x}}_{M F}^{(n-1)} \cdot \hat{\mathbf{x}}_{M F}^{(n-1)}} .
$$

The CG-based detection algorithm outperforms the NS-based detection scheme in terms of performance and complexity [30] [31]. The CG method requires a large number of iterations and include several divisions [30] [32].

\section{H. Optimized Coordinate Descent Method}

Coordinate descent (CD) obtains an approximate solution of a large number of convex optimization using series of simple, coordinate-wise updates. The estimated solution can be concluded as

$$
\hat{\mathbf{x}}_{k}=\left(\left\|\mathbf{h}_{k}\right\|^{2}+N_{0}\right)^{-1} \mathbf{h}_{k}^{H}\left(\mathbf{y}-\sum_{j \neq k} \mathbf{h}_{j} \mathbf{x}_{j}\right),
$$

where $N_{o}$ is the noise variance. In OCD, a preprocessing and algorithm restructuring will be performed to minimize the amount of operations during each iteration.

\section{Richardson Method}

It utilizes symmetric matrices defined as positive at their execution and can be slowed as it approaches the exact solution over time. In order to achieve a fast convergence, a relaxation parameter $\omega$ has been introduced into iterative process and it satisfies $0<\omega \leq \frac{2}{\lambda}$ where $\lambda$ is the largest eigenvalue of the symmetric positive definite matrix $\mathbf{H}$ [33]. Richardson iteration is described mathematically as

$$
\mathbf{x}^{(n+1)}=\mathbf{x}^{(n)}+\omega\left(\mathbf{y}-\mathbf{H} \mathbf{x}^{(n)}\right) \quad n=0,1,2, \cdots .
$$

The initial solution $\mathbf{x}^{(0)}$ can be identified as $2 K \times 1$ zero vector without loss of generality as no a priori knowledge of the final solution is available [34]. Richardson method requires a large number of iterations [33]. It also has uncertain relaxation parameter $\omega$ [32], [35]. Table I shows the comparison of computational complexity between several approximate matrix inversion methods. The complexity of all methods will be increased over iterations while the complexity of Neumann method will be increased significantly when $n \geq 3$.
Table I

COMPLEXITY COMPARISON AMONG APPROXIMATE MATRIX INVERSION METHODS

\begin{tabular}{|l|l|}
\hline Method & Number of multiplications \\
\hline Neumann & $4 N^{3}(n-2)+(2 K+1) K^{2}+(4 N-1) K$ \\
\hline Richardson & $(4 N+4 n) K^{2}+2 K N$ \\
\hline SOR & $(4 N+4 n-2) K^{2}+2(N-n+1) K$ \\
\hline GS & $(4 N+4 n-2) K^{2}+2(N-2 n+1) K$ \\
\hline OCD & $(8 N K+4 K) n$ \\
\hline Jacobi & $(4 N+4 n+1) K^{2}+2 \mathrm{NK}$ \\
\hline CG & $\left(N+2 K^{2}\right) n$ \\
\hline
\end{tabular}

\section{RESUlTS AND DiscUSSION}

In this section, the performances and the complexity figures of detectors based on the Neumann, GS, SOR, Jacobi, Richardson, OCD and CG will be validated. A comparison among the approximate matrix inversion methods will be provided in BER performance versus the SNR and number of multiplications. In all simulations, we consider the Rayleigh fading channel and the configuration of massive MIMO systems with users and BS antennas are $16 \times 32$ and $16 \times 128$ and the modulation scheme is 64QAM.

Figure 1 shows the BER performance of the MMSE algorithm and MMSE utilizing several approximate matrix inversion methods in $16 \times 128 \mathrm{MIMO}$ system. It also presents the BER performance when the number of iterations $(n)$ vary from 1 until 5. The BER performance of the mentioned methods improved when $n$ increased. However, when $n=1$, GS achieved the best BER performance while CG has the worst performance. In addition, GS, SOR and OCD achieved the MMSE performance when when $n=3$ while $\mathrm{CG}$ achieved the same performance when $n=4$. It is also clear that Richardson method outperforms the Neumann and Jacobi methods when $n \geq 4$. However, it is known that not every iteration improved the BER performance. For instance, the BER performance of GS, SOR, OCD are not improved when $n \geq 4$.

Figure 2 shows the comparison among the approximate matrix inversion methods in $16 \times 32$ where $\beta$ is closer to 1 . As compared with a MIMO system with a small $\beta$, it is clear that more iterations are required to achieve a near MMSE performance. For a small number of iterations $(n=1,2)$, the detector based on approximate matrix inversion methods provided a low performance. However, the performance of Neumann and Jacobi methods have not been improved over iterations while SOR outperforms the other methods for different iterations and it achieved a near MMSE performance when $n=6$.

The selection of $\omega$ plays a crucial role in achieving a good BER performance of the SOR and the Richardson based detectors. Figure 3 shows the BER performance of the MMSE signal detection utilizing the SOR algorithm versus $\omega$ at SNR $=10 \mathrm{~dB}$. The BER performance improved when $\omega$ increased while the best BER performance can be achieved when the value of $\omega$ is 0.9 then the performance starts to decrease for higher values for all iterations.

Sequentially, Fig. 4 illustrates the BER performance of the MMSE signal detection utilizing the Richardson method 


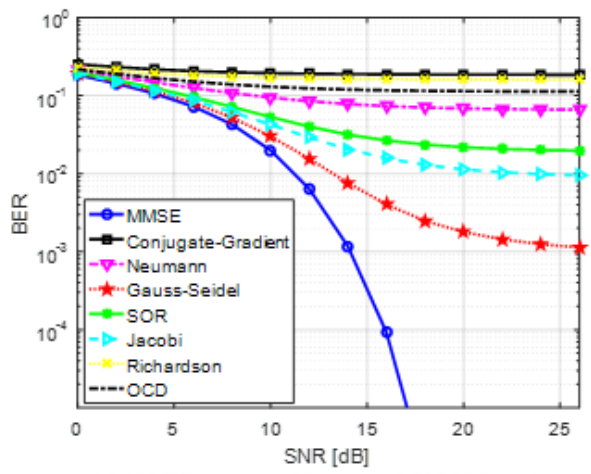

(a) $128 \mathrm{BS}$ antennas, 16 users and 64QAM, $\mathrm{n}=1$

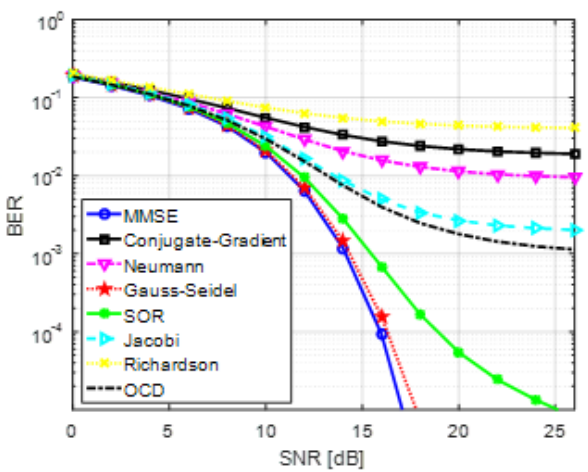

(b) $128 \mathrm{BS}$ antennas, 16 users and 64QAM, $\mathrm{n}=2$

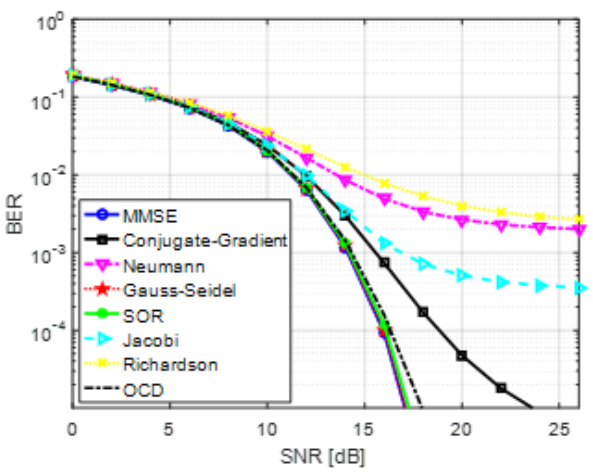

(c) $128 \mathrm{BS}$ antennas, 16 users and 64QAM, $\mathrm{n}=3$

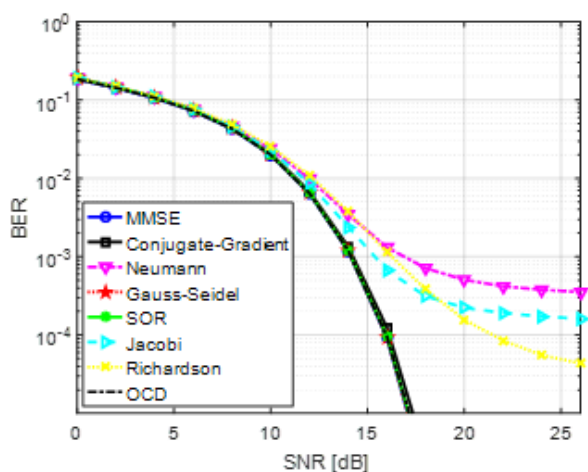

(d) $128 \mathrm{BS}$ antennas, 16 users and 64QAM, $\mathrm{n}=4$

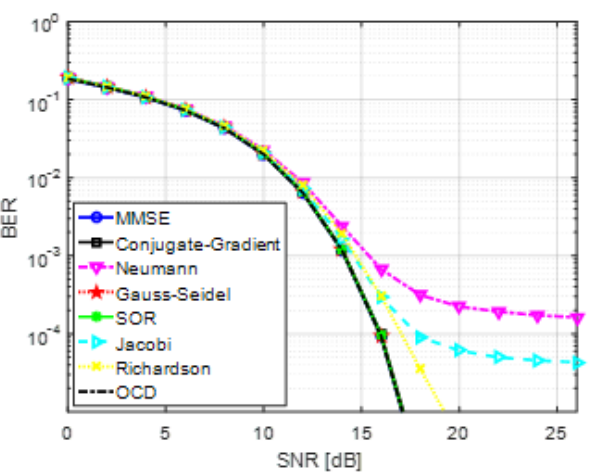

(e) $128 \mathrm{BS}$ antennas, 16 users and 64QAM, $\mathrm{n}=5$

Figure 1. Performances of different approximate detection methods and the exact MMSE for $16 \times 128$ massive MIMO system

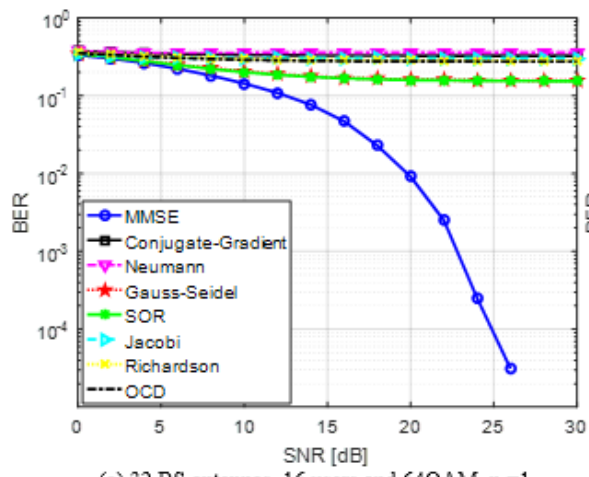

(a) 32 BS antennas, 16 users and 64QAM, $n=1$

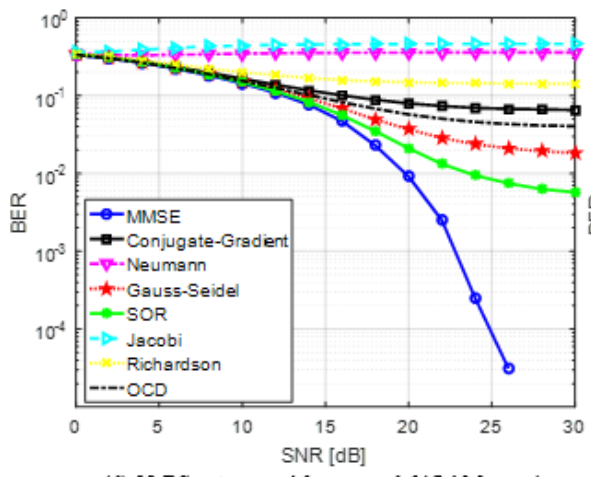

(d) $32 \mathrm{BS}$ antennas, 16 users and 640AM, $\mathrm{n}=4$

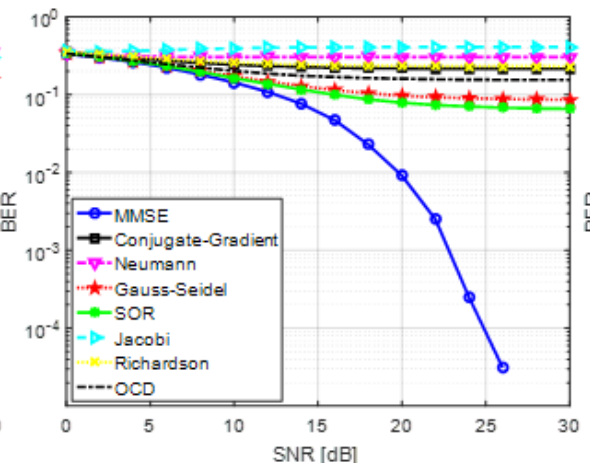

(b) 32 BS antennas, 16 users and 64QAM, $n=2$

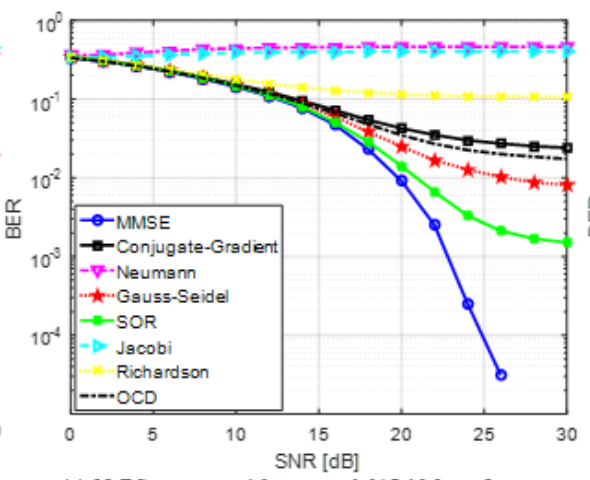

(e) 32 BS antennas, 16 users and 640AM, $n=5$

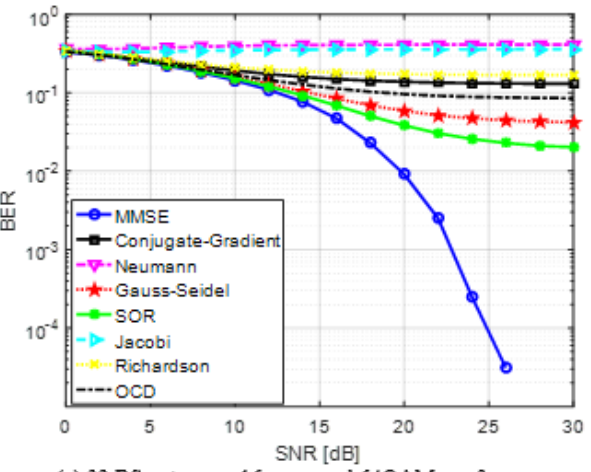

(c) $32 \mathrm{BS}$ antennas, 16 users and 64QAM, $\mathrm{n}=3$

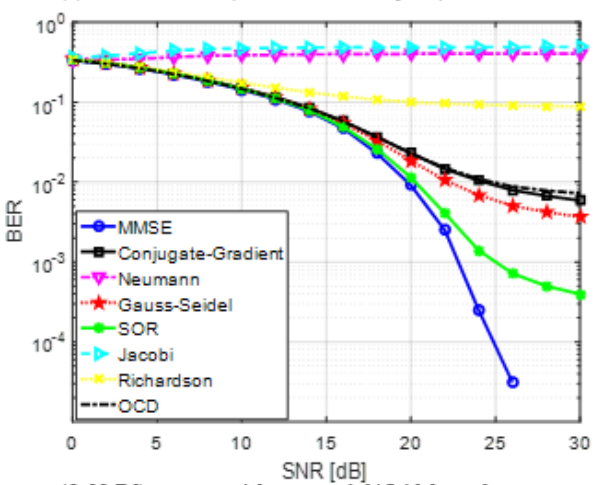

(f) $32 \mathrm{BS}$ antennas, 16 users and 640AM, $\mathrm{n}=6$

Figure 2. Performance of different approximate detection methods and the exact MMSE for $16 \times 32$ massive MIMO system 


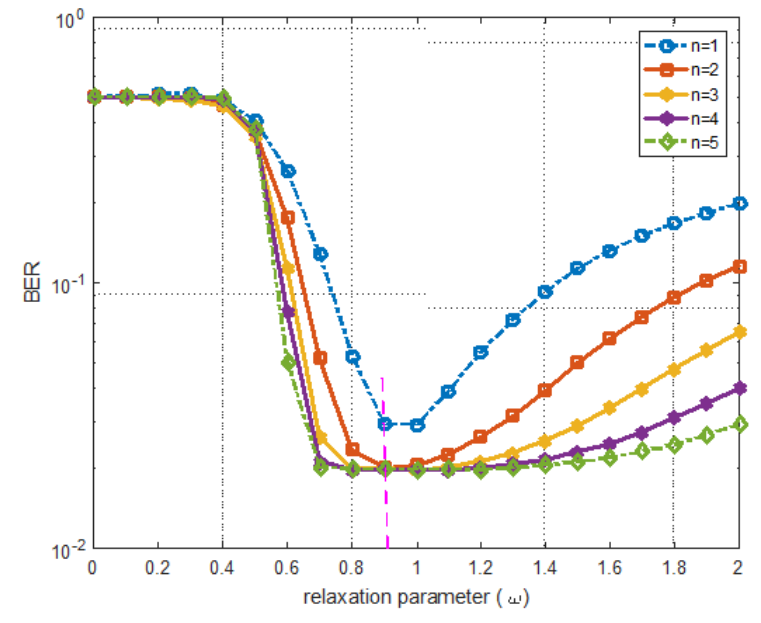

Figure 3. Performance of $16 \times 128$ MIMO with MMSE detector utilizing SOR versus $\omega$ with $\mathrm{SNR}=10 \mathrm{~dB}$

versus $\omega$ at $\mathrm{SNR}=10 \mathrm{~dB}$. The best performance have been achieved when $\omega=\frac{2}{\lambda}$.

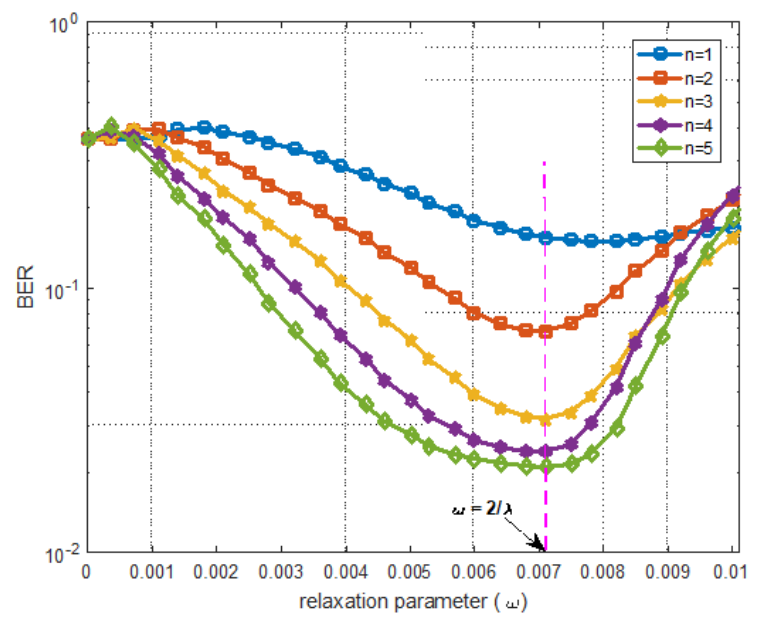

Figure 4. Performances of $16 \times 128$ MIMO with MMSE detector utilizing Richardson versus $\omega$ with SNR $=10 \mathrm{~dB}$

Figure 5 presents the complexity comparison in the number of multiplications in each iteration as mentioned in Table I. It is clear that detectors based on the Neumann, the CG and the OCD method has the lowest number of multiplications where OCD has the best BER performance among the mentioned methods (Fig. 1 and 2).

Figure 6 shows the required SNR, number of iterations, and the number of multiplication to achieve BER $=10^{-2}$. It is clear that the detector based on GS method achieves the BER target at SNR $=14 \mathrm{~dB}$ with $n=1$ and $12 \mathrm{~dB}$ with $n=2$. In addition, the detector based on Jacobi method achieves the BER target at $\mathrm{SNR}=22 \mathrm{~dB}$ with $n=1,14 \mathrm{~dB}$ with $n=2$, and $12 \mathrm{~dB}$ with $n=3$. Sequentially, the detector based on OCD and CG methods can achieve the BER $=10^{-2}$ at $12 \mathrm{~dB}$ with $n=3$ and a small number of multiplications.

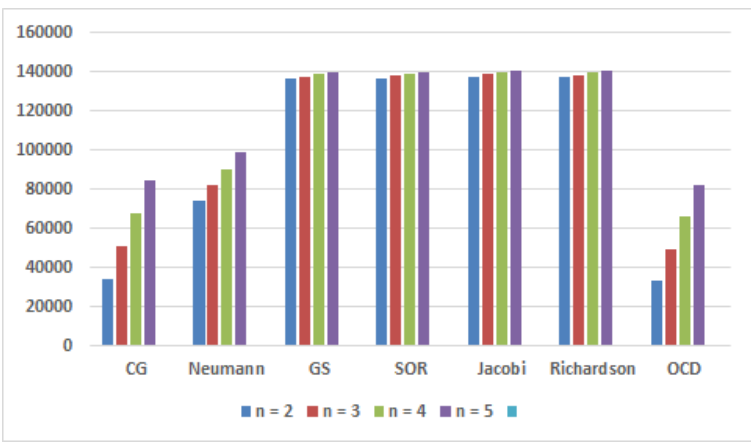

Figure 5. Complexity comparison among different approximate inversion methods in $16 \times 128$ MIMO

\section{CONCLUSION}

This paper has presented a comparison between the exact MMSE based MIMO detector and several approximate matrix inversion methods at different number of iterations. The simulation results show that the detector based on the GS method outperformed the other detector when $\beta$ is small. On the other hand, when $\beta$ is large, a detector based on the SOR method has achieved the best BER performance. The selection of the relaxation parameter $(\omega)$ has been studied for both SOR and Richardson methods. It has been shown that the optimum BER performance can be achieved when $\omega=\frac{2}{\lambda}$ in the Richardson method. Furthermore, this paper has compared the computational complexity of the approximate inversion methods. The Neumann method, the OCD method, and the CG method have achieved the lowest number of multiplications, while the CG method outperforms the Neumann and the OCD methods.

\section{ACKNOWLEDGMENT}

Authors would like to thank A'Sharqiyah University (ASU) for the conference travel grant.

\section{REFERENCES}

[1] C. VNI., "Cisco visual networking index: Global mobile data traffic forecast update, 2016-2021," white paper, 2017.

[2] M. A. M. Albreem and N. A. H. B. Ismail, "A review: detection techniques for lte system," Telecommunication Systems, vol. 63, no. 2, pp. 153-168, Oct 2016. [Online]. Available: https://doi.org/10.1007/s11235-015-0112-8

[3] M. A. M. Albreem, " $5 \mathrm{~g}$ wireless communication systems: Vision and challenges," in 2015 International Conference on Computer, Communications, and Control Technology (I4CT), April 2015, pp. 493-497.

[4] M. A. M. Albreem, A. El-Saleh, M. Isa, W. Salah, and M. Juso, "Green internet of things: an overivew," in 2017 4th IEEE International Conference on Smart Instrumentation, Measurement and Applications (ICSIMA2017), November 2017, pp. 1-6.

[5] Y. Jeon, C. Song, S. R. Lee, S. Maeng, J. Jung, and I. Lee, "New beamforming designs for joint spatial division and multiplexing in large-scale miso multi-user systems," IEEE Transactions on Wireless Communications, vol. 16, no. 5, pp. 3029-3041, May 2017.

[6] A. Zaib, M. Masood, A. Ali, W. Xu, and T. Y. Al-Naffouri, "Distributed channel estimation and pilot contamination analysis for massive mimoofdm systems," IEEE Transactions on Communications, vol. 64, no. 11, pp. 4607-4621, Nov 2016.

[7] A. A. Lu, X. Gao, Y. R. Zheng, and C. Xiao, "Low complexity polynomial expansion detector with deterministic equivalents of the moments of channel gram matrix for massive mimo uplink," IEEE Transactions on Communications, vol. 64, no. 2, pp. 586-600, Feb 2016. 


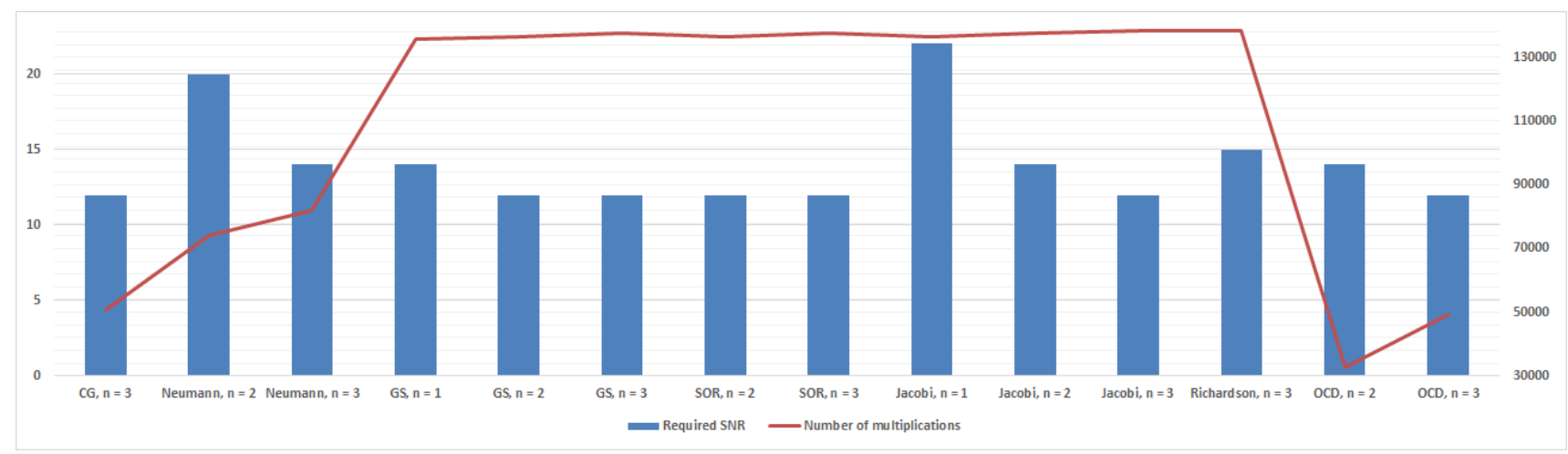

Figure 6. Performance-complexity trade-off to achieve BER $=10^{-2}$ in $16 \times 128 \mathrm{MIMO}$

[8] S. K. Mohammed, A. Chockalingam, and B. S. Rajan, "A lowcomplexity near-ml performance achieving algorithm for large mimo detection," in 2008 IEEE International Symposium on Information Theory, July 2008, pp. 2012-2016.

[9] Q. Zhou and X. Ma, "Element-based lattice reduction algorithms for large mimo detection," IEEE Journal on Selected Areas in Communications, vol. 31, no. 2, pp. 274-286, February 2013.

[10] A. Elghariani and M. Zoltowski, "Low complexity detection algorithms in large-scale mimo systems," IEEE Transactions on Wireless Communications, vol. 15, no. 3, pp. 1689-1702, March 2016.

[11] K. Nikitopoulos, D. Chatzipanagiotis, C. Jayawardena, and R. Tafazolli, "Multisphere: Massively parallel tree search for large sphere decoders," in 2016 IEEE Global Communications Conference (GLOBECOM), Dec 2016, pp. 1-6.

[12] A. K. Sah and A. K. Chaturvedi, "An mmp-based approach for detection in large mimo systems using sphere decoding," IEEE Wireless Coтmunications Letters, vol. 6, no. 2, pp. 158-161, April 2017.

[13] J. C. Chi, Y. C. Yeh, I. W. Lai, and Y. H. Huang, "Sphere decoding for spatial permutation modulation mimo systems," in 2017 IEEE International Conference on Communications (ICC), May 2017, pp. 18.

[14] P. Zhang, L. Liu, G. Peng, and S. Wei, "Large-scale mimo detection design and fpga implementations using sor method," in 2016 8th IEEE International Conference on Communication Software and Networks (ICCSN), June 2016, pp. 206-210.

[15] M. Mandloi, M. A. Hussain, and V. Bhatia, "An improved multiple feedback successive interference cancellation algorithm for mimo detection," in 2016 8th International Conference on Communication Systems and Networks (COMSNETS), Jan 2016, pp. 1-6.

[16] T. L. Narasimhan and A. Chockalingam, "Channel hardening-exploiting message passing (chemp) receiver in large mimo systems," in 2014 IEEE Wireless Communications and Networking Conference (WCNC), April 2014, pp. 815-820.

[17] S. Ghacham, M. Benjillali, and Z. Guennoun, "Low-complexity detection for massive mimo systems over correlated rician fading," in 2017 13th International Wireless Communications and Mobile Computing Conference (IWCMC), June 2017, pp. 1677-1682.

[18] J. Zhou, J. Hu, J. Chen, and S. He, "Biased mmse soft-output detection based on conjugate gradient in massive mimo," in 2015 IEEE 11th International Conference on ASIC (ASICON), Nov 2015, pp. 1-4.

[19] Z. Zhang, J. Wu, X. Ma, Y. Dong, Y. Wang, S. Chen, and X. Dai, "Reviews of recent progress on low-complexity linear detection via iterative algorithms for massive mimo systems," in 2016 IEEE/CIC International Conference on Communications in China (ICCC Workshops), July 2016, pp. 1-6.

[20] L. Dai, X. Gao, X. Su, S. Han, C. L. I, and Z. Wang, "Low-complexity soft-output signal detection based on gauss 2013; seidel method for uplink multiuser large-scale mimo systems," IEEE Transactions on Vehicular Technology, vol. 64, no. 10, pp. 4839-4845, Oct 2015.

[21] Z. Wu, C. Zhang, Y. Xue, S. Xu, and X. You, "Efficient architecture for soft-output massive mimo detection with gauss-seidel method," in 2016 IEEE International Symposium on Circuits and Systems (ISCAS), May 2016, pp. 1886-1889.
[22] E. Agrell, T. Eriksson, A. Vardy, and K. Zeger, "Closest point search in lattices," IEEE Transactions on Information Theory, vol. 48, no. 8, pp. 2201-2214, Aug 2002.

[23] E. N. Onggosanusi, A. G. Dabak, T. Schmidl, and T. Muharemovic, "Capacity analysis of frequency-selective mimo channels with suboptimal detectors," in 2002 IEEE International Conference on Acoustics, Speech, and Signal Processing, vol. 3, May 2002, pp. III-2369-III-2372.

[24] J. C. Chen, "A low complexity data detection algorithm for uplink multiuser massive mimo systems," IEEE Journal on Selected Areas in Communications, vol. 35, no. 8, pp. 1701-1714, Aug 2017.

[25] B. Kang, J. H. Yoon, and J. Park, "Low complexity massive mimo detection architecture based on neumann method," in 2015 International SoC Design Conference (ISOCC), Nov 2015, pp. 293-294.

[26] L. Fang, L. Xu, and D. D. Huang, "Low complexity iterative mmse-pic detection for medium-size massive mimo," IEEE Wireless Communications Letters, vol. 5, no. 1, pp. 108-111, Feb 2016.

[27] Z. Wu, Y. Xue, X. You, and C. Zhang, "Hardware efficient detection for massive mimo uplink with parallel gauss-seidel method," in 2017 22nd International Conference on Digital Signal Processing (DSP), Aug 2017, pp. $1-5$.

[28] W. Song, X. Chen, L. Wang, and X. Lu, "Joint conjugate gradient and jacobi iteration based low complexity precoding for massive mimo systems," in 2016 IEEE/CIC International Conference on Communications in China (ICCC), July 2016, pp. 1-5.

[29] J. Jin, Y. Xue, Y. L. Ueng, X. You, and C. Zhang, "A split preconditioned conjugate gradient method for massive mimo detection," in 2017 IEEE International Workshop on Signal Processing Systems (SiPS), Oct 2017, pp. 1-6.

[30] B. Yin, M. Wu, J. R. Cavallaro, and C. Studer, "Conjugate gradient-based soft-output detection and precoding in massive mimo systems," in 2014 IEEE Global Communications Conference, Dec 2014, pp. 3696-3701.

[31] Y. Hu, Z. Wang, X. Gaol, and J. Ning, "Low-complexity signal detection using cg method for uplink large-scale mimo systems," in 2014 IEEE International Conference on Communication Systems, Nov 2014, pp. $477-481$.

[32] L. Shao and Y. Zu, "Joint newton iteration and neumann series method of convegence-accelerating matrix inversion approximation in linear precoding for massive mimo systems," Mathematical Problems in Engineering, vol. 2016, 2016.

[33] X. Gao, L. Dai, Y. Ma, and Z. Wang, "Low-complexity near-optimal signal detection for uplink large-scale mimo systems," Electronics Letters, vol. 50, no. 18, pp. 1326-1328, August 2014.

[34] A. Bjorck, Numerical Methods for Least Squares Problems. Society for Industrial and Applied Mathematics, 1996. [Online]. Available: http://epubs.siam.org/doi/abs/10.1137/1.9781611971484

[35] H. Costa and V. Roda, "A scalable soft richardson method for detection in a massive mimo system," Przeglad Elektrotechniczny, vol. 92, no. 5, pp. 199-203, 2016. 